\title{
A REMARK ON THE RITT ORDER OF ENTIRE FUNCTIONS DEFINED BY DIRICHLET SERIES
}

\author{
A. G. AZPEITIA ${ }^{1}$
}

1. Let $f(s)(s=\sigma+i t)$ be an entire function defined by an everywhere absolutely convergent Dirichlet series

$$
\sum_{n=1}^{\infty} a_{n} \exp \left(\lambda_{n} s\right) \quad\left(0<\lambda_{n}<\lambda_{n+1} \rightarrow \infty\right) .
$$

The Ritt order is defined [1, p. 77] as

$$
\rho=\limsup _{\sigma=\infty}[\log \log M(\sigma) / \sigma]
$$

with

$$
M(\sigma)=\underset{-\infty<t<\infty}{\operatorname{l.u.b.}}|f(\sigma+i t)| .
$$

2. We will show that the Ritt theorem $[1$, p. 78], which gives the value of $\rho$ in terms of the sequences $\left\{\left|a_{n}\right|\right\}$ and $\left\{\lambda_{n}\right\}$ under the restriction $\lim \inf _{n=\infty}\left[\lambda_{n} / \log n\right]>0$, can be improved as follows:

Let $\rho_{1}$ be defined as

$$
\rho_{1}=\limsup _{n=\infty}\left[\lambda_{n} \log \lambda_{n} / \log \left|a_{n}\right|^{-1}\right] .
$$

We will prove the following:

THEOREM. If $\sum_{n=1}^{\infty} a_{n} \exp \left(\lambda_{n} s\right)$ is absolutely convergent for all $s$, then $\rho \geqq \rho_{1}$, and if, in addition, it is assumed that

$$
\lim _{n=\infty}\left[\lambda_{n} \log \lambda_{n} / \log n\right]=\infty
$$

then $\rho=\rho_{1}$.

Proof. With no changes, the first part of the proof given by Mandelbrojt [2, p. 217] shows that $\rho \geqq \rho_{1}$ if $\rho<\infty$, and the same conclusion obviously holds if $\rho=\infty$.

To completely establish the theorem it is now enough to prove that if (1) holds, then $\rho \leqq \rho_{1}$. This result can be obtained by a modification of the second part of Mandelbrojt's proof [2, p. 218], as follows.

Received by the editors October 25, 1960.

1 This work is part of a research supported by a grant from the National Science Foundation (NSF-G14142). 
We can assume $\rho_{1}<\infty$, since otherwise the conclusion is trivial. Then for every $\delta>0$ there exists $n_{0}=n_{0}(\delta)$ such that $\left|a_{n}\right|$ $\leqq \exp \left[-\lambda_{n} \log \lambda_{n} /\left(\rho_{1}+\delta\right)\right]$ for all $n \geqq n_{0}$. Therefore, for every $\sigma$

$$
\begin{aligned}
M(\sigma) \leqq & \sum_{n=1}^{n_{0}-1}\left|a_{n}\right| \exp \left(\lambda_{n} \sigma\right) \\
& +\sum_{n=n_{0}}^{\infty} \exp \left[-\lambda_{n} \log \lambda_{n} /\left(\rho_{1}+\delta\right)+\lambda_{n} \sigma\right]
\end{aligned}
$$

because the series involved in the formula is easily seen to be convergent, since from (1) it follows that for every $\epsilon>0$, the series $\sum_{n=1}^{\infty} \exp \left(-\epsilon \lambda_{n} \log \lambda_{n}\right)$ converges to a finite sum $\phi(\epsilon)$.

Now, for any choice of $\alpha \geqq 0$ and $\beta>0$ such that $\alpha+\beta=1 /(\rho+\delta)$ we have

$$
\begin{aligned}
M(\sigma) & \leqq \\
& \cdot \sum_{n=1}^{n_{0}-1}\left|a_{n}\right| \exp \left(\lambda_{n} \sigma\right)+\max _{n \geqq 1}\left[\exp \left(-\alpha \lambda_{n} \log \lambda_{n}+\lambda_{n} \sigma\right)\right] \\
& \leqq \sum_{n=1}^{n_{0}-1}\left|a_{n}\right| \exp \left(\lambda_{n} \sigma\right)+\phi(\beta) \max _{x \geqq 0} \exp \left(-\alpha x \log \lambda_{n}\right) \\
& =\sum_{n=1}^{n_{0}-1}\left|a_{n}\right| \exp \left(\lambda_{n} \sigma\right)+\phi(\beta) \exp \{\alpha \exp [(\sigma-\alpha) / \alpha]\}
\end{aligned}
$$

and consequently $\rho=\lim \sup _{\sigma=\infty}[\log \log M(\sigma) / \sigma] \leqq 1 / \alpha$, and since $\sup \alpha=1 /\left(\rho_{1}+\delta\right)$ and $\delta$ is arbitrarily small, it follows that $\rho \leqq \rho_{1}$ and the theorem is proved.

Finally, it should be noticed that the assumption of the sequence $\left\{\lambda_{n}\right\}$ being monotonic, implies that (1) holds if and only if the series $\sum \exp \left(-\epsilon \lambda_{n} \log \lambda_{n}\right)$ converges for every $\epsilon>0$, and therefore the previous theorem is the best possible result that can be obtained by the present method of proof.

\section{REFERENCES}

1. J. F. Ritt, On certain points of the theory of Dirichlet series, Amer. J. Math. vol. 50 (1928) pp. 73-86.

2. S. Mandelbrojt, Dirichlet series, The Rice Institute Pamphlet, vol. 31, no. 4, October 1944, pp. 159-272.

UNIVERSITY OF MASSACHUSETTS 